\title{
Kerr scattering coefficients via isomonodromy
}

\author{
Bruno Carneiro da Cunha ${ }^{a}$ and Fábio Novaes ${ }^{b}$ \\ ${ }^{a}$ Departamento de Física, Universidade Federal de Pernambuco, \\ 50670-901, Recife, Pernambuco, Brazil \\ ${ }^{b}$ International Institute of Physics, Federal University of Rio Grande do Norte, \\ Av. Odilon Gomes de Lima 1722, Capim Macio, Natal-RN 59078-400, Brazil \\ E-mail: bcunha@df.ufpe.br, fabio.nsantos@gmail.com
}

ABSTRACT: We study the scattering of a massless scalar field in a generic Kerr background. Using a particular gauge choice based on the current conservation of the radial equation, we give a generic formula for the scattering coefficient in terms of the composite monodromy parameter $\sigma$ between the inner and the outer horizons. Using the isomonodromy flow, we calculate $\sigma$ exactly in terms of the Painlevé $\mathrm{V} \tau$-function. We also show that the eigenvalue problem for the angular equation (spheroidal harmonics) can be calculated using the same techniques. We use recent developments relating the Painlevé $\mathrm{V} \tau$-function to Liouville irregular conformal blocks to claim that this scattering problem is solved in the combinatorial sense, with known expressions for the $\tau$-function near the critical points.

KeYwords: Black Holes, Integrable Equations in Physics, Conformal and W Symmetry

ArXiv ePrint: 1506.06588 


\section{Contents}

1 Introduction 1

2 Klein-Gordon in Kerr 2

3 The Fuchsian system 3

4 The isomonodromy method $\quad 6$

4.1 Asymptotic wavefunctions and normalization 8

5 Angular equation $\quad 9$

6 Asymptotics of Painlevé V 10

7 Discussion $\quad 12$

\section{Introduction}

Black hole scattering theory is an important problem from the general relativity and string theory perspective. It serves as a way of testing linear stability [1], superradiance [2], black hole entropy [3] and relaxation times in AdS-CFT [4]. For the rich story behind it see $[5,6]$. Scattering is also important to study astrophysical problems related to the detection of gravitational waves and a possible mechanism for short gamma ray bursts, for example. See the introduction of [7], references therein and $[8,9]$ for discussions. It is also a daunting problem from the mathematical side, involving unknown properties of new special functions [10]. Most of the previous study in four dimensions on the subject relied on numerical analysis, either by direct numerical integration or by expansion of the solution in terms of known special functions [11-13]. Also, decoupling limits like the near-extremal cases were taken directly on the metric (see [14] for applications in Kerr-CFT).

In this letter we build on previous analytical work, a reverse Hilbert-Riemann problem [15], based on isomonodromy equations [16] to relate the scattering coefficients for a massless scalar field in four-dimensional Kerr background in terms of the Painlevé $\mathrm{V}$ transcendent $\tau$-function [17] and show that the problem can be solved in the combinatorial sense using the expasions of the $\tau$-function near the critical points. We also show that the angular equation can be solved implicitly using the same techniques, in a new way of extracting eigenvalues for the spheroidal wave functions $[18,19]$. The results shown here can be understood as the natural conclusion to the application of the monodromy problem to solve scattering problems as applied to black holes [20-23]. The result follows from a careful consideration of the "linearization" of the Painlevé V equation in the sense given by Miwa, Jimbo et al. in [24-26], and of the time-reversal symmetry. These two points 
allows us to place boundary conditions such that the two linearly independent solutions of the radial equation correspond to the two solutions involved in the connection problem of the Painlevé V equation [27]. Recent developments on the relation between Painlevé transcendents and the AGT conjecture $[28,29]$ show the striking connection between black holes and black hole scattering to Conformal Field Theory [30].

This letter is organized as follows. In section 2 we treat the radial equation for the massless Klein-Gordon field in a generic four-dimensional Kerr background. We write the equation in a canonical confluent Heun type and discuss the time-reversal symmetry. In section 3 we discuss how the scattering problem for the radial equation is connected to the connection problem of the confluent Heun equation. We describe the (extended) monodromy data of the confluent Heun equation, including the Stokes parameters, and use the time-reversal symmetry to parametrize the scattering coefficient in terms of the monodromy parameters. In section 4 we discuss how to extract these parameters from the Painlevé $\mathrm{V} \tau$-function, using the isomonodromy flow. In section 5 , we outline a procedure to extract the eigenvalues for the angular equation using the same structure. Finally, in section 6 we list the results of [17] which give a combinatorial expansion for the Painlevé $\mathrm{V} \tau$-function near $t_{0}=0$, which would correspond to our extremal limit. We close with a short discussion and future directions in section 7 .

\section{Klein-Gordon in Kerr}

It is known that the massless Klein-Gordon equation in a Kerr background is separable [31], $i$. e., the solution can be written as $\Phi\left(x^{\mu}\right)=e^{-i \omega t} e^{i m \phi} R(r) S(\theta)$. The angular part $S(\theta)$ is solved in terms of oblate scalar spheroidal harmonics, whose eigenvalues (the separation constant $C_{\ell, m}$ ) can be approximated for different asymptotic regimes, including low and high frequency limits $[18,32]$, and will be the object of study in section 5 . We will focus for now on the radial part

$$
\partial_{r}\left(Q(r) \partial_{r} R(r)\right)+\left(-C_{\ell, m}+\frac{W_{r}^{2}}{Q(r)}\right) R(r)=0,
$$

where $Q(r)$ and $W_{r}^{2}$ are given by

$$
\begin{aligned}
Q(r) & =r^{2}-2 M r+a^{2}=\left(r-r_{+}\right)\left(r-r_{-}\right), \\
W_{r} & =\omega\left(r^{2}+a^{2}\right)-a m .
\end{aligned}
$$

The form which will be useful for the analysis has

$$
z=2 i \omega\left(r-r_{-}\right), \quad \text { and } \quad y(z)=\left(r-r_{-}\right)^{\theta_{0} / 2}\left(r-r_{+}\right)^{\theta_{t_{0}} / 2} R(r),
$$

with the parameter

$$
t_{0}=2 i \omega\left(r_{+}-r_{-}\right) .
$$


The radial equation is written in this parametrization as

$$
\begin{gathered}
\frac{d^{2} y}{d z^{2}}+p(z) \frac{d y}{d z}+q(z) y(z)=0 \\
p(z)=\frac{1-\theta_{t_{0}}}{z-t_{0}}+\frac{1-\theta_{0}}{z}, \quad q(z)=-\frac{1}{4}+\frac{c_{0}}{z}+\frac{c_{t_{0}}}{z-t_{0}},
\end{gathered}
$$

which is known as the confluent Heun equation, with parameters given by

$$
\begin{gathered}
\theta_{0}=2 i \frac{\omega\left(r_{-}^{2}+a^{2}\right)-a m}{r_{-}-r_{+}}=-\frac{i}{2 \pi} \frac{\omega-\Omega m}{T_{-}}, \\
\theta_{t_{0}}=2 i \frac{\omega\left(r_{+}^{2}+a^{2}\right)-a m}{r_{+}-r_{-}}=\frac{i}{2 \pi} \frac{\omega-\Omega m}{T_{+}}, \\
t_{0} c_{0}=C_{\ell, m}+\frac{1}{2}\left(\left(1-t_{0}\right) \theta_{0}+\theta_{t_{0}}\right), \quad t_{0} c_{t_{0}}
\end{gathered}
$$

where we defined the temperatures $T_{ \pm}$and angular velocities $\Omega_{ \pm}$for the outer and inner horizons. For future reference, we also define

$$
\theta_{\infty}=1+2\left(c_{0}+c_{t_{0}}\right)=1-4 i M \omega=1-\theta_{0}-\theta_{t_{0}} .
$$

Also, eq. (2.1) has real coefficients, so if $R(r)$ is a solution, so is $R(r)^{*}$. We then define the radiation flux as

$$
j=-i Q(r)\left[R(r)^{*} \partial_{r} R(r)-R(r) \partial_{r} R(r)^{*}\right] .
$$

One should bear in mind that real $r$ means $z^{*}=-z$, because of $(2.3)$.

\section{The Fuchsian system}

Writing the solutions for (2.5) in terms of Frobenius expansions at $z=0$ or $z=t_{0}$ is straightforward. On the other hand, extracting the scattering coefficients from the solutions is not straightforward in the sense that not enough information is known about the function defined by the ODE. To wit, we are interested in the so-called connection problem: how a solution of (2.5) with known behavior at one singular point, say $y_{t_{0}}(z)$ at $z=t_{0}$, is related to the pair of solutions with known behavior at another singular point, say, $y_{\infty}^{ \pm}(z)$ at $z=\infty$. So, for example, in

$$
y_{t_{0}}(z)=C y_{\infty}^{+}(z)+D y_{\infty}^{-}(z)
$$

$C$ and $D$ are the connection coefficients. If $y_{t_{0}}(z)$ represents a purely ingoing wave at the outer horizon $z=t_{0}$ and $y_{\infty}^{ \pm}(z)$ ingoing and outgoing waves at infinity, then the connection coefficients are indeed the scattering coefficients. As we will see below, solving the connection problem allows us to solve the scattering problem.

In [16], the authors proposed a method based on the Schlesinger equations to extract the scattering coefficients. It relies on viewing the ODE (2.5) as the equation satisfied by the first row of the $2 \times 2$ matricial Fuchsian system

$$
\frac{d}{d z} \Phi(z)=A(z) \Phi(z)
$$


and to extract the connection coefficients from the monodromy data of the Fuchsian system. The monodromy data for the system at hand is defined as follows: suppose $A(z)$ is meromorphic and let $\left\{z_{i}\right\}$ be the isolated singular points of the system (3.2). The point at $z=\infty$ is studied by the substitution $u=1 / z$. The solution $\Phi(z)$ will also cease to be analytic at these points and behave singularly there. Since $\tilde{\Phi}(z)=\Phi\left(e^{2 \pi i}\left(z-z_{i}\right)+z_{i}\right)$ is also a solution of (3.2), then we must have $\tilde{\Phi}(z)=\Phi(z) M_{i}$, with $M_{i}$ a constant matrix associated with a circuit around $z_{i}$ - the monodromy.

The form of $M_{i}$ depends on the behavior of $A(z)$ near the singular point $z_{i}$. If $A(z)$ diverges like $\left(z-z_{i}\right)^{-1}$, then the singular point is called regular and $M_{i}$ is conjugated to $\operatorname{diag}\left(e^{2 \pi i \alpha_{i}^{+}}, e^{2 \pi i \alpha_{i}^{-}}\right)$, where $\alpha_{i}^{ \pm}$are the solutions of the indicial equation near $z_{i}$. This form of $M_{i}$ stems from the leading behavior $\left(z-z_{i}\right)^{\alpha_{i}^{ \pm}}$of the solution near $z_{i}$, a branch point for $\Phi(z)$. We will for now on assume that the differences $\alpha_{i}^{+}-\alpha_{i}^{-}$are not integers for the radial equation. If, however, $A(z)$ diverges like $\left(z-z_{i}\right)^{-1-r_{i}}$, where the integer $r_{i}>0$ is the Poincaré rank, the solution will have an essential singularity and will experience the Stokes phenomenon: the leading term in the Frobenius expansion displays an exponential behavior which diverges or converges depending on which direction the limit $z \rightarrow z_{i}$ is taken in the complex plane. In order to describe it, we will specialize to our case. In equation (2.5), the matricial system has $A(z)$ with the structure

$$
A(z)=\frac{1}{2} \sigma_{3}+\frac{A_{0}}{z}+\frac{A_{t}}{z-t},
$$

where there are two regular singular points, at $z=0$ and $z=t$, and an irregular point at $z=\infty$ with Poincaré rank 1. We also have the usual Pauli matrix $\sigma_{3}$. The elements of $A_{0}$ and $A_{t}$ in (3.3) are independent of $z$ and are obtained from the ODE (2.5). This identification has extra freedom because the Fuchsian system has more free parameters than the ODE. We thus pick the gauge choice

$$
\operatorname{Tr} A_{i}=\theta_{i}, \quad \operatorname{det} A_{i}=0, \quad \operatorname{Tr} \sigma_{3}\left(A_{0}+A_{t}\right)=-\theta_{\infty},
$$

which, along with the ODE parameters (2.6), fixes the elements of $A_{i}$ up to conjugation by $e^{u \sigma_{3}}$. The epithet gauge choice will become clear in the next section.

In order to describe the system at the irregular singular point $z=\infty$, one defines the sectors $[27,33]$

$$
\mathcal{S}_{j}=\left\{z \in \mathbb{C} \mid(2 j-5) \frac{\pi}{2}<\arg z<(2 j-1) \frac{\pi}{2}\right\},
$$

for $j \in \mathbb{Z}$. On each $\mathcal{S}_{j}$, one defines the asymptotic behavior for $\Psi(z)=z^{-\frac{1}{2}\left(\theta_{0}+\theta_{t}\right)} \Phi(z)$ based on the gauge choice (3.4) for $A(z)$,

$$
\left.\Psi(z)\right|_{j}=\left(\mathbb{1}+\mathcal{O}\left(z^{-1}\right)\right) \exp \left(\frac{1}{2} z \sigma_{3}\right) z^{-\frac{1}{2} \theta_{\infty} \sigma_{3}} .
$$

These canonical solutions are connected by the Stokes matrices $\Psi_{j+1}(z)=\Psi_{j}(z) S_{j}$ and, given that $\Psi_{j+2}\left(e^{2 \pi i} z\right)=\Psi_{j}(z) e^{-\pi i \theta_{\infty} \sigma_{3}}$, we have $S_{j+2}=e^{\pi i \theta_{\infty} \sigma_{3}} S_{j} e^{-\pi i \theta_{\infty} \sigma_{3}}$, and the Stokes matrices are all determined by any consecutive 2 , which have the following structure

$$
S_{2 j}=\left(\begin{array}{cc}
1 & s_{2 j} \\
0 & 1
\end{array}\right), \quad S_{2 j+1}=\left(\begin{array}{cc}
1 & 0 \\
s_{2 j+1} & 1
\end{array}\right)
$$


and the $s_{2 j}$ and $s_{2 j+1}$ are called Stokes parameters. Local solutions of the Fuchsian system at $z=\infty$ are defined in the Riemann surface of the logarithm, and these parameters describe how the solution (3.6) changes as one changes the leaf of the surface. The (extended) monodromy data for (2.5) is completely determined by the monodromy matrices $M_{0}$ and $M_{t}$, at the regular singular points 0 and $t$, respectively, and the Stokes matrices $S_{0}$ and $S_{1}$, which determine the (formal) monodromy matrix at $\infty$ in sector $\mathcal{S}_{j}$ by

$$
\left.M_{\infty}\right|_{\mathcal{S}_{j}}=S_{j} S_{j+1} e^{\pi i \theta_{\infty} \sigma_{3}} .
$$

Knowledge of the $\theta_{i}$ and of two consecutive Stokes parameters, say, $s_{1}$ and $s_{2}$ is sufficient data for solving the connection problem. We define the connection matrices $E_{i}$ by

$$
M_{i}=E_{i}^{-1}\left(\begin{array}{cc}
e^{i \pi \theta_{i}} & 0 \\
0 & e^{-i \pi \theta_{i}}
\end{array}\right) E_{i}, \quad i=0,1,
$$

and those are given in terms of $\theta_{i}, \sigma, s_{1}$ and $s_{2}$ in [27,33]. Note that, because $M_{\infty} M_{t_{0}} M_{0}=$ $\mathbb{1}$, we have that the combined monodromy parameter $2 \cos \pi \sigma=\operatorname{Tr} M_{0} M_{t}$ satisifies

$$
2 \cos \pi \sigma=2 \cos \pi \theta_{\infty}+e^{i \pi \theta_{\infty}} s_{1} s_{2},
$$

and that $M_{\infty}=E_{\infty}^{-1} e^{i \pi \sigma \sigma_{3}} E_{\infty}$. The connection matrices $E_{i}$ can be seen - when properly normalized by $\operatorname{det} E_{i}=1$ - as the matrices that implement the change to the "natural basis" of solutions of the ODE at $z_{i}$ where the monodromy $M_{i}$ is diagonal. Explicit formulas for $E_{i}$ in terms of monodromy data are given in [33]. We use the remaining gauge symmetry $A(z) \rightarrow e^{u \sigma_{3}} A(z) e^{-u \sigma_{3}}$ so that both entries in the first row of $\Phi(z)$ have equal but opposite current density normalization (2.8), defined as $j^{ \pm}$. So we have the entries $[\Phi(z)]_{11}=u_{\infty}^{+} \propto$ $R_{\infty}^{+}$and $[\Phi(z)]_{12}=u_{\infty}^{-} \propto R_{\infty}^{-}$being the normalized incoming and outgoing waves near infinity. Because the $j^{ \pm}$are conserved, this choice also means that the entries will be normalized near the outer horizon $t_{0}$, up to a phase. Therefore, the entries of the connection matrix

$$
E_{\infty t_{0}}=E_{\infty} E_{t_{0}}^{-1}
$$

will give the explicit scattering coefficients, allowing us to write $\mathcal{T}$ and $\mathcal{R}$ such that

$$
u_{t}^{-}=\frac{1}{\mathcal{T}} u_{\infty}^{+}+\frac{\mathcal{R}}{\mathcal{T}} u_{\infty}^{-}
$$

and by construction the time-reversed wave is equal, up to a phase, to the entry $[\Phi(z)]_{12}$ near $t_{0}$. We notice that Det $E_{\infty t_{0}}=1$ implies $|\mathcal{R}|^{2}=1-|\mathcal{T}|^{2}$, which is the condition of flux conservation. Now, by substitution of the diagonalized forms of the monodromy matrices in $\operatorname{Tr} M_{\infty} M_{t}=\operatorname{Tr} M_{0}^{-1}$, and using (3.12), we obtain the formula

$$
|\mathcal{T}|^{2}=\left|\frac{\sin \pi \sigma \sin \pi \theta_{t_{0}}}{\sin \frac{\pi}{2}\left(\theta_{0}+\sigma-\theta_{t_{0}}\right) \sin \frac{\pi}{2}\left(\theta_{0}-\sigma+\theta_{t_{0}}\right)}\right|,
$$

which gives the coefficient in terms of the composite monodromy parameter $\sigma$. In order to compute it, we will need the structure of isomonodromy deformations, outlined in the next section. After embedding this system in the isomonodromy formulation, we will be able to give explicit coordinate dependence of the wave functions. 


\section{The isomonodromy method}

The idea of considering the Fuchsian system (3.2) instead of the scalar ODE came in the context of the Riemann-Hilbert problem, since a simple counting argument [35] shows that the generic scalar ODE does not have enough parameters to realize all possible monodromy matrices. The Fuchsian system, however, has too many parameters, so there is a family of $A(z)$ in (3.2) with the same monodromy data. The description of this family can be understood physically: suppose we are given an invertible solution $\Phi(z)$ of (3.2) with some singular points. Then we can understand $A(z)=\left[\frac{d}{d z} \Phi(z)\right] \Phi(z)^{-1}$ as a "gauge potential" describing non-abelian charges, dipoles or multipoles at each singular point. The physical configuration described by the gauge potential is invariant under generic gauge transformations $A(z) \rightarrow U(z) A(z) U(z)^{-1}+\left[\frac{d}{d z} U(z)\right] U(z)^{-1}$, meaning that the monodromy data (non-abelian holonomies) are also invariant under these transformations.

In the case at hand (2.5), with $A(z)$ of the form (3.3), we supply a $t$-component to this gauge potential,

$$
B(z)=-\frac{A_{t}}{z-t}
$$

in such a way that the "field strength" $\partial_{t} A-\partial_{z} B+[A, B]$ is zero if the Schlesinger equations hold

$$
\frac{\partial A_{0}}{\partial t}=\frac{1}{t}\left[A_{t}, A_{0}\right], \quad \frac{\partial A_{t}}{\partial t}=-\frac{1}{t}\left[A_{t}, A_{0}\right]-\frac{1}{2}\left[A_{t}, \sigma_{3}\right] .
$$

The monodromy data of the Fuchsian system (3.2) will be independent of $t$ if $A_{0}(t)$ and $A_{t}(t)$ in (3.3) satisfy the equations above (4.2). For the relationship between the Schlesinger equations and the theory of flat holomorphic connections we refer to [24-26, 35].

The system (3.2) has some direct conserved charges. The gauge choice (3.4) is justified now because these are the constant of motions under Schlesinger evolution. With these constraints, the equation satisfied by any element of the first row of $\Phi(z)$ in (3.2), with $A(z)$ given by (3.3), is of the form

$$
\begin{gathered}
\frac{d^{2} y}{d z^{2}}+p(z) \frac{d y}{d z}+q(z) y=0, \\
p(z)=\frac{1-\theta_{0}}{z}+\frac{1-\theta_{t}}{z-t}-\frac{1}{z-\lambda}, \quad q(z)=-\frac{1}{4}+\frac{C_{0}}{z}+\frac{C_{t}}{z-t}+\frac{\mu}{z-\lambda},
\end{gathered}
$$

where $C_{0}, C_{t}, \lambda$ and $\mu$ are functions of the entries of $A(z)$. Compared to (2.5), the system above has an extra singularity at $z=\lambda$. However, this is an apparent one - its monodromy is trivial, as the indicial equation has exponents 0 and 2 and there is no logarithm behavior due to the relation between $\mu, \lambda$ and $t$,

$$
\mu^{2}-\left[\frac{\theta_{0}-1}{\lambda}+\frac{\theta_{t}-1}{\lambda-t}\right] \mu+\frac{C_{0}}{\lambda}+\frac{C_{t}}{\lambda-t}=\frac{1}{4} .
$$

The system (4.2) is better known in another clothing, as

$$
y(t)=\frac{\left[A_{0}(t)\right]_{11}\left[A_{t}(t)\right]_{12}}{\left[A_{t}(t)\right]_{11}\left[A_{0}(t)\right]_{12}}=\frac{\theta_{0}+\theta_{t}-\theta_{\infty}-(2 \mu-1)(\lambda-t)}{\theta_{0}+\theta_{t}-\theta_{\infty}-(2 \mu-1) \lambda}
$$


can be checked to satisfy the Painlevé V equation. These are part of the Painlevé transcendents family of differential equations: non-linear second order differential equations whose solutions do not possess movable branch points [35]. These define new special functions, with applications in integrable systems, random matrix theory and conformal field theory - see $[17,36]$ for references in those applications. In [27], asymptotic expressions for the Painlevé $\mathrm{V}$ system were given in the guise of the $\tau$-function

$$
\begin{aligned}
\frac{d}{d t} \log \tau\left(t,\left\{\theta_{i}\right\}, s_{1}, s_{2}\right) & =-\frac{1}{2} \operatorname{Tr} \sigma_{3} A_{t}-\frac{1}{t} \operatorname{Tr} A_{0} A_{t} \\
& =-\frac{\lambda(\lambda-t)}{t}\left[\mu^{2}-\left(\frac{\theta_{0}}{\lambda}+\frac{\theta_{t}}{\lambda-t}\right) \mu+\frac{\theta_{\infty}}{2 \lambda}-\frac{1}{4}\right]-\frac{\theta_{0} \theta_{t}}{t},
\end{aligned}
$$

in terms of the monodromy data. The $\tau$-function is the most natural isomonodromy invariant that can be defined, with a clear interpretation as a generating functional in quantum field theory applications [37-39].

As the problem now stands, knowledge of the $\tau$ function solves the system completely. We pick suitable initial conditions for $\mu$ and $\lambda$ in (4.3), and from those recover the $A_{0}$ and $A_{t}$, which then will set the initial conditions for the $\tau$ function. By choosing the initial conditions

$$
\theta_{t}=\theta_{t_{0}}-1, \quad \lambda\left(t_{0}\right)=t_{0}, \quad \mu\left(t_{0}\right)=\frac{c_{t_{0}}}{\theta_{t_{0}}-1},
$$

based on the parameters of the initial Heun equation (2.6) we have

$$
\begin{aligned}
\left.t \frac{d}{d t} \log \tau\left(t ;\left\{\theta_{i}\right\}, s_{i}\right)\right|_{t=t_{0}} & =t_{0} c_{t_{0}}-\theta_{0}\left(\theta_{t_{0}}-1\right), \\
\left.t \frac{d}{d t}\left(t \frac{d}{d t} \log \tau\left(t ;\left\{\theta_{i}\right\}, s_{i}\right)\right)\right|_{t=t_{0}} & =t_{0} \frac{\theta_{t_{0}}-1}{2}
\end{aligned}
$$

which can be formally inverted to yield the Stokes parameters $s_{1}$ and $s_{2}$, and hence $\sigma$. We note that our definition (4.6) differs slightly from the one in [27] by

$$
\tau_{\text {ours }}(t)=t^{\left(\left(\theta_{0}-\theta_{t}\right)^{2}-2 \theta_{\infty}^{2}\right) / 4}\left[\tau_{\text {Jimbo }}(t)\right]^{-1} \text {. }
$$

One also notes that (4.8) allows us to interpret the $\tau$-function as the generating function that implements the canonical transformation between the non-trivial monodromy data $s_{1}, s_{2}$ and the canonically conjugated parameters of the ODE $t_{0}, c_{t_{0}}$.

The structure outlined here gives another interpretation for the $\tau$-function. One of the most fundamental results from Miwa, Jimbo et al. on the Schlesinger system [24-26] — see also [35] — was the Hamiltonian structure of the isomonodromy flow. This structure stems from the symplectic structure of flat holomorphic connections (see [16] for a description), but can be intuitively understood from the algebraic constraint (4.4). One first notes that $C_{0}$ and $C_{t}$ are not independent, they are related to the asymptotic behavior at $z=\infty$, via $\theta_{\infty}$,

$$
C_{0}+C_{t}=-\mu+\frac{\theta_{\infty}-1}{2} .
$$

Varying $t$ will now tie the variation of $\lambda$ and $\mu$ in a symplectic system, in which $C_{0}$ can be thought of as the Hamiltonian function. Now, by "solving" the Hamiltonian system one 
means giving a canonical transformation from the coordinates $C_{0}, t_{0}$ to coordinates where the isomonodronic flow is trivial. But the latter is parametrized by the monodromy data, which by construction is invariant under the flow. The $\tau$-function is then the generating function that implements this canonical transformation, as evidenced by (4.8). In [40] a set of Darboux coordinates were constructed for the Painlevé VI case. It would be interesting to give a parallel of that construction to the case at hand.

\subsection{Asymptotic wavefunctions and normalization}

Now, with the embedding of the radial equation (2.1) in the matricial system (3.2) via (4.7), we can work out the specific form of the wave functions in terms of the coordinate $r$. The form is not necessary for our results, but will help comparing with previous work $[12,13]$. The normalization of the elementary matrix $\Phi(z)$ is given by

$$
\operatorname{det} \Phi(z)=z^{\theta_{0}}\left(z-t_{0}\right)^{\theta_{t_{0}}-1}
$$

with boundary conditions at $z=\infty$ given by (3.6). It is straightforward to verify that the Wronskian between the two entries of the first row is given by:

$$
W(z)=\left[\Phi^{\prime}(z)\right]_{11}[\Phi(z)]_{12}-\left[\Phi^{\prime}(z)\right]_{12}[\Phi(z)]_{11}=[A(z)]_{12} \operatorname{det} \Phi .
$$

Now, using the form of $[A(z)]_{12}$ compatible with (4.3), with conditions (4.7):

$$
[A(z)]_{12}=\frac{k(z-\lambda)}{z(z-t)}=\frac{k}{z}
$$

we arrive at:

$$
W(z)=k z^{\theta_{0}-1}\left(z-t_{0}\right)^{\theta_{t_{0}}-1} .
$$

We remind the reader that the value $k$ is "gauge dependent", and proceed to normalize the asymptotic from the entries of $\Phi(z)$. From (3.6), we have:

$$
\begin{aligned}
& {\left[\Phi_{\infty}(z)\right]_{11}=z^{\frac{1}{2}\left(\theta_{0}+\theta_{t_{0}}-1-\theta_{\infty}\right)} e^{\frac{1}{2} z}\left(1+\mathcal{O}\left(z^{-1}\right)\right),} \\
& {\left[\Phi_{\infty}(z)\right]_{12}=k z^{\frac{1}{2}\left(\theta_{0}+\theta_{t_{0}}-1+\theta_{\infty}\right)-1} e^{-\frac{1}{2} z}\left(1+\mathcal{O}\left(z^{-1}\right)\right),}
\end{aligned}
$$

where the limit is taken through the direction $\arg z=\frac{1}{2} \pi(z \rightarrow+i \infty)$. Following [27] we define the Frobenius basis at $z=t_{0}$ to be:

$$
\Phi_{t_{0}}(z)=G_{t_{0}}\left(\begin{array}{cc}
1 & 0 \\
0 & \left(z-t_{0}\right)^{\theta_{t_{0}}}-1
\end{array}\right)\left(\mathbb{1}+\mathcal{O}\left(z-t_{0}\right)\right),
$$

where $G_{t_{0}}$ is a matrix which diagonalizes $A_{t}$. Its particular form will not be important for us save that for the conditions (4.7) it can be checked to be of the lower triangular form. From this we can write the Wronskian-normalized basis for wavefunctions at $z=t_{0}$ :

$$
\left[\Phi_{t_{0}}(z)\right]_{11}=t_{0}^{\frac{1}{2} \theta_{0}}\left(1+\mathcal{O}\left(z-t_{0}\right)\right), \quad\left[\Phi_{t_{0}}(z)\right]_{12}=k t_{0}^{\frac{1}{2} \theta_{0}-1}\left(z-t_{0}\right)^{\theta_{t_{0}}}\left(1+\mathcal{O}\left(z-t_{0}\right)\right),
$$


Now, we can write the current-normalized wavefunctions in terms of the coordinate $r$ :

$$
\begin{aligned}
& u_{\infty}^{+}=i(2 \omega)^{\frac{1}{2}-2 i M \omega} e^{2 \pi M \omega} R_{\infty}^{+}(r)=\frac{1}{\sqrt{2 \omega}} \frac{1}{r} e^{i \omega\left(r-r_{-}+2 M \log \left(r-r_{-}\right)\right)}\left(1+\mathcal{O}\left(r^{-1}\right)\right), \\
& u_{\infty}^{-}=\frac{i}{k}(2 \omega)^{\frac{1}{2}+2 i M \omega} e^{-2 \pi M \omega} R_{\infty}^{-}(r)=\frac{1}{\sqrt{2 \omega}} \frac{1}{r} e^{-i \omega\left(r-r_{-}+2 M \log \left(r-r_{-}\right)\right)}\left(1+\mathcal{O}\left(r^{-1}\right)\right), \\
& u_{t_{0}}^{+}=\frac{1}{k} e^{-i \frac{\pi}{2} \theta_{0}}\left|\frac{2 \omega t_{0}}{\theta_{t_{0}}}\right|^{\frac{1}{2}} R_{t_{0}}^{+}(r)=-i \frac{(2 \omega)^{\frac{1}{2}+2 i M \omega}}{\left|t_{0} \theta_{t_{0}}\right|^{\frac{1}{2}}}\left(r-r_{+}\right)^{\frac{1}{2} \theta_{t_{0}}}\left(1+\mathcal{O}\left(r-r_{+}\right)\right) \\
& u_{t_{0}}^{-}=e^{-i \frac{\pi}{2} \theta_{0}}\left|\frac{2 \omega}{t_{0} \theta_{t_{0}}}\right|^{\frac{1}{2}} R_{t_{0}}^{-}(r)=\frac{(2 \omega)^{\frac{1}{2}\left(1+\theta_{0}\right)}}{\left|t_{0} \theta_{t_{0}}\right|^{\frac{1}{2}}}\left(r-r_{+}\right)^{-\frac{1}{2} \theta_{t_{0}}}\left(1+\mathcal{O}\left(r-r_{+}\right)\right) .
\end{aligned}
$$

As anticipated in the previous section, a suitable choice of $k$ can make the relative normalizations equal. The result (3.13) follows. In order to implement the time-reversal procedure, one has to check the relative phases and include them explicitly in the formula for the connection coefficients. At any rate, the basis at $r=r_{+}$is different from the one considered by $[12,13]$, which were mainly concerned about generic spin Teukolsky equation and did not work the normalization out explicitly near $r=r_{+}$. In the non-zero spin case, one has to solve not only for the monodromy but also for the Teukolsky-Starobinski identities [41]. It is an interesting future problem to try and extend the results presented here to that case.

\section{Angular equation}

The general solution for the composite monodromy parameter $\sigma$ using (4.8) (and (3.10)) can of course be applied to more generic situations. The associated equation for the angular part of the wavefunction $S(\theta)$ is also of the confluent Heun type. Following [19], the scalar spheroidal harmonics are the solutions of the angular equation:

$$
\frac{d}{d x}\left[\left(1-x^{2}\right) \frac{d}{d x} S_{\ell, m}\right]+\left[-(a \omega)^{2}\left(1-x^{2}\right)+C_{\ell, m}+2 a m \omega-\frac{m^{2}}{1-x^{2}}\right] S_{\ell, m}=0,
$$

for which the solution has the following behavior near the singular points $x=\cos \theta= \pm 1$ :

$$
S_{\ell, m}=\left\{\begin{array}{l}
(1+x)^{m / 2}, \quad x \rightarrow-1, \\
(1-x)^{-m / 2}, \quad x \rightarrow+1,
\end{array}\right.
$$

for integer $m$. It is clear that this behavior will only happen for particular discrete values of the constant $C_{\ell, m}$, which can be fed into (2.1).

To bring this equation into the canonical confluent Heun form (2.5), we make:

$$
z=2 a \omega(x-1), \quad y(z)=(1+x)^{m / 2}(1-x)^{-m / 2} S_{\ell, m}(x),
$$

and the new parameters are:

$$
t_{0}=-4 a \omega, \quad \theta_{0}=-\theta_{t_{0}}=-m, \quad c_{0}=-c_{t_{0}}=\frac{C_{\ell, m}+a^{2} \omega^{2}}{4 a \omega} .
$$

Now, from the discussion above, the existence of a solution of the confluent Heun equation with behavior given by (5.2) puts constraints on the connection matrices. In this particular 
case, one of the natural solutions at $z=0$ will not "mix" with the natural solution at $z=1$. This would correspond to the vanishing of one of the constants at (3.1). One should not that the second natural solution will of course mix. Also, since the parameter $\theta_{0}$ and $\theta_{t}$ are integers, there will be logarithm behavior for the other solution - in the case $\omega \rightarrow 0$ it would correspond to the associated Legendre functions of the second kind.

It is not hard to see that from this condition the composite monodromy $2 \cos \pi \sigma=$ $\operatorname{Tr} M_{0} M_{t}$ will have to be special:

$$
\sigma=2 \ell, \quad \ell \in \mathbb{Z}
$$

as required by the vanishing of the analogue of (3.13) for this case. Now, using (4.8), one has a - somewhat formal - solution for the eigenvalues:

$$
C_{\ell, m}=-a^{2} \omega^{2}-m(m-1)+\left.t \frac{d}{d t} \log \tau(t ; m,-m, 2 \ell)\right|_{t=-4 a \omega} .
$$

Since $\tau(t)$ is also a function of the Stokes parameters $s_{1}, s_{2}$, the second equation in (4.8) is also necessary to find the $C_{\ell}$.

\section{Asymptotics of Painlevé V}

In the spirit of making the analysis self-contained, we copy the relevant formulae about the Painlevé V $\tau$-function from [17]. In the following we use their definition for the $\tau$-function:

$$
\tau(t)=t^{\left(\theta_{0}-\theta_{t}\right)^{2} / 4}[\tilde{\tau}(t)]^{-1},
$$

and assume $\theta_{i}, \sigma$ and $\theta_{i}-\theta_{j}, \sigma-\theta_{i}$ are not integers. The expansion for the tau-function is of the form

$$
\tilde{\tau}(t, \vec{\theta})=\sum_{n \in \mathbb{Z}} C\left(\left\{\theta_{i}\right\}, \frac{1}{2} \sigma+n\right) \hat{s}^{n} t^{\left(\frac{1}{2} \sigma+n\right)^{2}} \mathcal{B}\left(\left\{\theta_{i}\right\}, \frac{1}{2} \sigma+n ; t\right),
$$

where the irregular conformal block $\mathcal{B}$ is given as a power series over the set of Young tableaux $\checkmark$ :

$$
\mathcal{B}\left(\left\{\theta_{i}\right\}, \frac{1}{2} \sigma ; t\right)=e^{-\frac{1}{2} \theta_{t} t} \sum_{\lambda, \mu \in \mathbb{Y}} \mathcal{B}_{\lambda, \mu}\left(\left\{\theta_{i}\right\}, \frac{1}{2} \sigma\right) t^{|\lambda|+|\mu|},
$$

with coefficients

$$
\begin{aligned}
\mathcal{B}_{\lambda, \mu}=\prod_{(i, j) \in \lambda} \frac{\left(-\frac{1}{2} \theta_{\infty}+\frac{1}{2} \sigma+i-j\right)\left(\left(\frac{1}{2} \theta_{t}+\frac{1}{2} \sigma+i-j\right)^{2}-\frac{1}{4} \theta_{0}^{2}\right)}{h_{\lambda}^{2}(i, j)\left(\lambda_{j}^{\prime}+\mu_{i}-i-j+1+\sigma\right)} \times \\
\times \prod_{(i, j) \in \mu} \frac{\left(-\frac{1}{2} \theta_{\infty}-\frac{1}{2} \sigma+i-j\right)\left(\left(\frac{1}{2} \theta_{t}-\frac{1}{2} \sigma+i-j\right)^{2}-\frac{1}{4} \theta_{0}^{2}\right)}{h_{\mu}^{2}(i, j)\left(\lambda_{i}+\mu_{j}^{\prime}-i-j+1+\sigma\right)},
\end{aligned}
$$

where $\lambda$ denotes a Young tableau, $\lambda_{i}$ is the number of boxes in row $i, \lambda_{j}^{\prime}$ is the number of boxes in column $j$ and $h_{\lambda}(i, j)=\lambda_{i}+\lambda_{j}^{\prime}-i-j+1$ is the hook length related to the box 
$(i, j) \in \lambda$. The structure constants $C$ are rational products of Barnes functions

$$
C\left(\left\{\theta_{i}\right\}, \sigma\right)=\prod_{\epsilon= \pm} \frac{G\left(1-\frac{1}{2} \theta_{\infty}+\epsilon \frac{1}{2} \sigma\right) G\left(1+\frac{1}{2} \theta_{t}+\frac{1}{2} \theta_{0}+\epsilon \frac{1}{2} \sigma\right) G\left(1+\frac{1}{2} \theta_{t}-\frac{1}{2} \theta_{0}+\epsilon \frac{1}{2} \sigma\right)}{G(1+\epsilon \sigma)},
$$

where $G(z)$ is defined by the functional equation $G(1+z)=\Gamma(z) G(z)$. The parameters $\sigma$ and $s$ in (6.2) are related to the "constants of integration" of the Painlevé V equation. In our treatment, they are functions of the Stokes parameters $s_{1}$ and $s_{2}$. The parameter $\sigma$ is given by (3.10), whereas the expression for $\hat{s}$ in (6.2) is rather long and involved. We will outline the procedure in section 10 of [33] to compute it. Let $\hat{M}_{0}, \hat{M}_{t}$ be the monodromy matrices for the hypergeometric equations. They are of the form $\hat{M}_{i}=\hat{E}_{i}^{-1} e^{i \pi \theta_{i} \sigma_{3}} \hat{E}_{i}$ with $\hat{E}_{i}$ given by equation (10.15) in [33]. They satisfy $\hat{M}_{t} \hat{M}_{0}=e^{-i \pi\left(\theta_{0}+\theta_{1}\right)} \operatorname{diag}\left(e^{-i \pi \sigma}, e^{i \pi \sigma}\right)$. We introduce the matrix

$$
S=\left(\begin{array}{cc}
-e^{-i \pi\left(\sigma+\theta_{\infty}\right) / 2} \frac{\Gamma(-\sigma)}{\Gamma\left(1-\frac{1}{2}\left(\sigma-\theta_{\infty}\right)\right)} & \frac{\Gamma(-\sigma)}{\Gamma\left(1-\frac{1}{2}\left(\sigma+\theta_{\infty}\right)\right)} \\
e^{i \pi\left(\sigma-\theta_{\infty}\right) / 2} \frac{\Gamma(\sigma)}{\Gamma\left(\frac{1}{2}\left(\sigma+\theta_{\infty}\right)\right)} & \frac{\Gamma(-\sigma)}{\Gamma\left(\frac{1}{2}\left(\sigma-\theta_{\infty}\right)\right)}
\end{array}\right)
$$

whose purpose is to match the diagonal monodromy of the hypergeometric system at infinity to the non-diagonal monodromy of the confluent hypergeometric system at the origin. It is defined up to a diagonal matrix $s^{-\sigma_{3}}$. We now have the monodromy parametrization for $E_{i}$ in $(3.9)$ :

$$
E_{i}=\hat{E}_{i} s^{-\sigma_{3}} S R^{-\sigma_{3}},
$$

where $R^{2}=s_{1} / s_{2}$ serves to bring $M_{\infty}$ to a symmetric form. With this intricate construction, the parameter $s$ can be read implicitly from $M_{t}=M_{\infty}^{-1} M_{0}^{-1}$ - or more easily from its trace. The parameter $\hat{s}$ in (6.2) is given by $\hat{s}=-\frac{1}{2}\left(\sigma+\theta_{\infty}\right) s^{2}$. These expressions are purely algebraic in the sense that they can be computed from the constraints $M_{\infty} M_{t} M_{0}=\mathbb{1}$ obeyed by the monodromy matrices. The expansion (6.2) is obtained from the AGT conjecture [28], relating the Liouville conformal blocks to the instanton partition function of $\mathcal{N}=2$ SUSY quiver theories in four dimensions via a confluence limit.

The asymptotic limit of the Painlevé $\mathrm{V} \tau$-function was also considered in great detail in $[27,33]$. For our purposes, in the limit $t_{0} \rightarrow 0$, the sum (6.2) can be approximated by three terms:

$$
\begin{aligned}
\tilde{\tau}\left(t,\left\{\theta_{i}\right\}, \sigma\right) & =e^{-\frac{1}{2} \theta_{t} t} t^{(\sigma-2 n)^{2} / 4} f(t), \\
f(t) & =K\left(1+B_{1} t+C_{1} t^{1-(\sigma-2 n)}+\mathcal{O}\left(t^{2}, t^{2(1 \pm(\sigma-2 n))}\right)\right),
\end{aligned}
$$

where we assume that $2 n<\sigma<2 n+2$. The value of $n \in \mathbb{Z}$ will be obtained below, and we will also assume that $\varrho=\sigma-2 n<1$, which can be accomplished by shifting $n$, and the calculation that ensues will be analogous as below. As per (6.2) the leading parameters of the expansion of $f(t)$ are:

$$
\begin{gathered}
K=C\left(\left\{\theta_{i}\right\}, \frac{1}{2} \sigma-n\right), \quad B_{1}=-\frac{\theta_{\infty}}{4 \varrho^{2}}\left(\theta_{t}^{2}-\theta_{0}^{2}+\varrho^{2}\right), \\
C_{1}=\frac{\left(\theta_{\infty}+\varrho\right)}{8 \varrho^{2}(1-\varrho)^{2}}\left(\left(\theta_{t}+\varrho\right)^{2}-\theta_{0}^{2}\right) \hat{s}^{-1} .
\end{gathered}
$$


Now, (4.8) is written as:

$$
\begin{aligned}
\frac{1}{4}\left(\left(\theta_{0}-\theta_{t_{0}}+1\right)^{2}-\varrho^{2}\right)-t_{0} \frac{\dot{f}}{f}+\frac{1}{2} t_{0}\left(\theta_{t_{0}}-1\right) & =t_{0} c_{t_{0}}-\theta_{0}\left(\theta_{t_{0}}-1\right) \\
-t_{0} \frac{\dot{f}}{f}-t_{0}^{2} \frac{\ddot{f}}{f}+t_{0}^{2} \frac{\dot{f}^{2}}{f^{2}} & =0 .
\end{aligned}
$$

As alluded above, the second equation does not entail information about the initial value of the isomonodromy flow beyond the value of $t_{0}$. We will see it as fixing the value of $\hat{s}$ in terms of $\varrho$. Plugging the expansion for $f(t)$ as above, we find:

$$
C_{1}=-\frac{B_{1}}{(1-\varrho)^{2}} t^{\varrho}+\mathcal{O}\left(t^{2 \varrho}\right)
$$

Now, back to (4.8) and isolating $\varrho$, we find:

$$
\varrho^{2}=\left(\theta_{0}+\theta_{t_{0}}\right)^{2}-1+4 C_{\ell, m}-2 t_{0}+4 t_{0} \theta_{t_{0}}+4 t_{0} \frac{\varrho}{1-\varrho} B_{1}+\mathcal{O}\left(t^{1+\epsilon}\right)
$$

where $\epsilon=\min (\varrho, 1-\varrho)$. To first order in $t_{0}$, we then have:

$$
\begin{aligned}
& \varrho^{2} \simeq \varrho_{0}^{2}-t_{0}\left[2-4 \theta_{t_{0}}-\frac{\theta_{\infty}}{\varrho_{0}\left(1-\varrho_{0}\right)}\left(\left(\theta_{t_{0}}-1\right)^{2}-\theta_{0}^{2}+\varrho_{0}^{2}\right)\right], \\
& \varrho_{0}^{2}=-1-16 M^{2} \omega^{2}+4 C_{\ell, m},
\end{aligned}
$$

where we see that the integer $n$ is chosen to offset the integer part of $C_{\ell, m}$. Also, for small $\omega, \varrho_{0}$ is purely imaginary, but the $t_{0}$ corrections will introduce a real part, and the expansion will have poor convergence. This is verified for the Painlevé VI expansions using formulas analogue to (6.2) in [17], as convergence of the series improves for finite $t_{0}$. Notwithstanding these remarks, we find qualitative agreement with $[12,15]$ even though the small $t_{0}$ expansion is not strictly speaking a low-frequency expansion, as other parameters also depend on $\omega$.

At any rate, the expression (4.8) should be valid for generic values of the parameters, and with the results of section 5 where the separation constant $C_{\ell, m}$ are also computed using isomonodromy, we can claim the formal analytical solution of the scattering problem is presented. We leave for future work a survey of the values for the scattering coefficients, as well as expansions for the near extremal and near-superradiance $\theta_{0} \approx 0$ cases.

\section{Discussion}

In this letter, we sucessfully related the scattering coefficients for a massless scalar field in a Kerr background in terms of the Painlevé V $\tau$-function. By choosing the current normalization of the ingoing and outgoing waves, we first establish that the expression (3.13) does give the transmission coefficient directly in terms of the composite monodromy parameter $\sigma$. Also, by relating the problem of finding $\sigma$ to the inverse Riemann-Hilbert problem, we are able to give analytic, if implicit solutions for the composite monodromy (4.8) and the eigenvalue of the angular equation (5.6) in terms of the Painlevé V $\tau$-function. Also, 
approximate expressions for small isomonodromy parameter - related to low-frequency and/or near extremal case - can be obtained from the asymptotics of Painlevé V equation in terms of more elementary functions. It is our hope that the application of the techniques outlined here and in previous work by the authors [16] will help solve many outstanding problems in scattering for black-hole backgrounds, like Kerr-(A)dS, and higher spin perturbations. It should be stressed that the usefulness of (4.8) goes far beyond the low-energy or the near horizon expansions, - both tied to small $t_{0}$ - allowing one to study normal modes, and the onset of superradiance at $\theta_{0} \approx 0$. One can also use essentially the same arguments given here to study scalar perturbations between the inner and outer horizon, providing yet another tool for testing for instabilities. We will leave these important directions to future work.

The result showed here relied on the conservation of current argument, which allowed us to equate the scattering problem to the (mathematically defined) connection problem of the radial equation. Although this needs proof, the same argument should work for arbitrary spin perturbations, the so-called Teukolsky Master Equation (TME), whose radial and angular equations for Kerr background are also separable and yield the same confluent Heun type considered here. This should provide a powerful analytical tool to deal with normal modes, generation of gravitational waves by black holes, and the linear stability of the inner horizon.

In a more abstract viewpoint, the results extracted here point to the striking relationship between black hole physics and Conformal Field Theory. Relations for the $\tau$ function (6.2) were obtained because of recents results concerning Liouville Field Theories in the semi-classical limit, where the Painlevé V $\tau$-function arises as the expansion of the irregular conformal blocks, which are obtained from a confluence limit of (normalized) 4-point functions in Liouville Field Theory (see [17] and [16] for details). The 4-point function itself arises as the Painlevé VI $\tau$-function, and in the Black Hole case in the Kerr-de Sitter conformally coupled scalar field scattering [42]. The confluence limit of the Painlevé VI is the same as the zero cosmological constant limit. This can be seen somehow as an extension of the ideas of [30], where conformal symmetry was also used to determine Black Hole scattering in the extreme Kerr limit. As it happens, the correspondence seems exact, at least for a wide class of (vacuum and charged) Black Holes in four dimensions.

While this seems surprising at first glance, one cannot help but wonder whether this fact is yet another facet on the intricate relationship between integrable systems and Conformal Field Theory [43]. General Relativity solutions like the (static, axisymmetric) black hole systems consist an integrable sector of Einstein equations in four dimensions [44]. Requisition that the solutions of this integrable sector are singularity free basically imposes that there is a coordinate patch where the components of the metric are given in terms of rational functions. It follows then, apart from the non-trivial fact of separability of the equations, that the differential equations governing the propagation of fields in these backgrounds have its singularities easily classified, being Fuchsian or having a higher Poincaré index. Moreover, the isomonodromy flow provides a non-linear symmetry of these equations stemming from the theory of flat holomorphic connections and the Painlevé property. All in all, everything conspires to have the conformal blocks "solving" Fuchsian-type of differ- 
ential equations, since conformal blocks are in principle extracted from the representation theory of the Virasoro algebra. We can then describe the chain of deep mathematical facts pointing to the relationship between four-dimensional black holes and Conformal Field Theory. It is a very interesting open problem to see if this holds in the generic case.

\section{Acknowledgments}

The authors would like to thank Marc Casals, Remo Ruffini, Monica Guica, Amílcar de Queiroz and A. P. Balachandran for useful discussions and comments. FN acknowledges partial support from $\mathrm{CNPq}$ and $\mathrm{BCdC}$ from PROPESQ/UFPE.

Open Access. This article is distributed under the terms of the Creative Commons Attribution License (CC-BY 4.0), which permits any use, distribution and reproduction in any medium, provided the original author(s) and source are credited.

\section{References}

[1] M. Dafermos and I. Rodnianski, Lectures on black holes and linear waves, Clay Math. Proc. 17 (2013) 97 [arXiv: 0811.0354] [InSPIRE].

[2] W.H. Press and S.A. Teukolsky, Floating orbits, superradiant scattering and the black-hole bomb, Nature 238 (1972) 211 [INSPIRE].

[3] J.W. York, Jr., Dynamical origin of black hole radiance, Phys. Rev. D 28 (1983) 2929 [INSPIRE].

[4] G.T. Horowitz and V.E. Hubeny, Quasinormal modes of AdS black holes and the approach to thermal equilibrium, Phys. Rev. D 62 (2000) 024027 [hep-th/9909056] [INSPIRE].

[5] K.D. Kokkotas and B.G. Schmidt, Quasinormal modes of stars and black holes, Living Rev. Rel. 2 (1999) 2 [gr-qc/9909058] [INSPIRE].

[6] E. Berti, V. Cardoso and A.O. Starinets, Quasinormal modes of black holes and black branes, Class. Quant. Grav. 26 (2009) 163001 [arXiv:0905.2975] [INSPIRE].

[7] S.R. Dolan, Scattering and absorption of gravitational plane waves by rotating black holes, Class. Quant. Grav. 25 (2008) 235002 [arXiv:0801.3805] [INSPIRE].

[8] P.P. Fiziev and D.R. Staicova, A new model of the central engine of GRB and the cosmic jets, Bulg. Astron. J. 11 (2009) 3 [arXiv: 0902. 2408] [INSPIRE].

[9] P. Kumar and B. Zhang, The physics of gamma-ray bursts \& relativistic jets, Phys. Rept. 561 (2014) 1 [arXiv:1410.0679] [InSPIRE].

[10] R.S. Borissov and P.P. Fiziev, Exact solutions of Teukolsky master equation with continuous spectrum, Bulg. J. Phys. 37 (2010) 065 [arXiv:0903.3617] [inSPIRE].

[11] E.W. Leaver, An analytic representation for the quasi normal modes of Kerr black holes, Proc. Roy. Soc. Lond. A 402 (1985) 285 [InSPIRE].

[12] S. Mano, H. Suzuki and E. Takasugi, Analytic solutions of the Teukolsky equation and their low frequency expansions, Prog. Theor. Phys. 95 (1996) 1079 [gr-qc/9603020] [INSPIRE].

[13] S. Mano and E. Takasugi, Analytic solutions of the Teukolsky equation and their properties, Prog. Theor. Phys. 97 (1997) 213 [gr-qc/9611014] [inSPIRE]. 
[14] G. Compere, The Kerr/CFT correspondence and its extensions: a comprehensive review, Living Rev. Rel. 15 (2012) 11 [arXiv:1203.3561] [INSPIRE].

[15] A. Castro, J.M. Lapan, A. Maloney and M.J. Rodriguez, Black hole scattering from monodromy, Class. Quant. Grav. 30 (2013) 165005 [arXiv:1304.3781] [InSPIRE].

[16] F. Novaes and B. Carneiro da Cunha, Isomonodromy, Painlevé transcendents and scattering off of black holes, JHEP 07 (2014) 132 [arXiv: 1404.5188] [INSPIRE].

[17] O. Gamayun, N. Iorgov and O. Lisovyy, How instanton combinatorics solves Painlevé VI, V and IIIs, J. Phys. A 46 (2013) 335203 [arXiv:1302.1832] [InSPIRE].

[18] C. Flammer, Spheroidal wave functions, Stanford University Press, Stanford CA U.S.A. (1957).

[19] E. Berti, V. Cardoso and M. Casals, Eigenvalues and eigenfunctions of spin-weighted spheroidal harmonics in four and higher dimensions, Phys. Rev. D 73 (2006) 024013 [Erratum ibid. D 73 (2006) 109902] [gr-qc/0511111] [INSPIRE].

[20] A. Neitzke, Greybody factors at large imaginary frequencies, hep-th/0304080 [INSPIRE].

[21] N. Andersson and C.J. Howls, The asymptotic quasinormal mode spectrum of nonrotating black holes, Class. Quant. Grav. 21 (2004) 1623 [gr-qc/0307020] [INSPIRE].

[22] L. Motl and A. Neitzke, Asymptotic black hole quasinormal frequencies, Adv. Theor. Math. Phys. 7 (2003) 307 [hep-th/0301173] [InSPIRE].

[23] U. Keshet and A. Neitzke, Asymptotic spectroscopy of rotating black holes, Phys. Rev. D 78 (2008) 044006 [arXiv:0709.1532] [InSPIRE].

[24] M. Jimbo, T. Miwa and A.K. Ueno, Monodromy preserving deformation of linear ordinary differential equations with rational coefficients, I, Physica D 2 (1981) 306.

[25] M. Jimbo and T. Miwa, Monodromy preserving deformation of linear ordinary differential equations with rational coefficients, II, Physica D 2 (1981) 407.

[26] M. Jimbo and T. Miwa, Monodromy preserving deformation of linear ordinary differential equations with rational coefficients, III, Physica D 4 (1981) 26.

[27] M. Jimbo, Monodromy problem and the boundary condition for some Painlevé equations, Publ. Res. Inst. Math. Sci. 18 (1982) 1137.

[28] L.F. Alday, D. Gaiotto and Y. Tachikawa, Liouville correlation functions from four-dimensional gauge theories, Lett. Math. Phys. 91 (2010) 167 [arXiv:0906.3219] [INSPIRE].

[29] V.A. Alba, V.A. Fateev, A.V. Litvinov and G.M. Tarnopolskiy, On combinatorial expansion of the conformal blocks arising from AGT conjecture, Lett. Math. Phys. 98 (2011) 33 [arXiv: 1012.1312] [INSPIRE].

[30] I. Bredberg, T. Hartman, W. Song and A. Strominger, Black hole superradiance from Kerr/CFT, JHEP 04 (2010) 019 [arXiv:0907.3477] [InSPIRE].

[31] B. Carter, Hamilton-Jacobi and Schrödinger separable solutions of Einstein's equations, Commun. Math. Phys. 10 (1968) 280 [InSPIRE].

[32] H. Volkmer, Spheroidal wave functions, http://dlmf.nist.gov/30.

[33] F. Andreev and A.V. Kitaev, Connection formulas for asymptotics of the fifth Painlevé transcendent on the real axis, Nonlinearity 13 (2000) 1801. 
[34] L.D. Landau and E.M. Lifshitz, Quantum mechanics (non-relativistic theory), in Course of Theoretical Physics, volume 3, $3^{\text {rd }}$ edition, Butterworth-Heinemann, Oxford U.K. (1997).

[35] K. Iwasaki, H. Kimura, S. Shimomura and M. Yoshida, From Gauss to Painlevé: a modern theory of special functions, Aspects of Mathematics E 16, Braunschweig Germany (1991).

[36] O. Gamayun, N. Iorgov and O. Lisovyy, Conformal field theory of Painlevé VI, JHEP 10 (2012) 038 [Erratum ibid. 10 (2012) 183] [arXiv:1207.0787] [INSPIRE].

[37] M. Jimbo, T. Miwa, Y. Môri and M. Sato, Density matrix of an impenetrable Bose gas and the fifth Painlevé transcendent, Physica D 1 (1980) 80.

[38] G. Segal and G. Wilson, Loop groups and equations of KdV type, Publ. Math. IHÉS 61 (1985) 5.

[39] L.J. Mason, M.A. Singer and N.M.J. Woodhouse, Tau functions and the twistor theory of integrable systems, J. Geom. Phys. 32 (2000) 397.

[40] N. Nekrasov, A. Rosly and S. Shatashvili, Darboux coordinates, Yang-Yang functional and gauge theory, Nucl. Phys. Proc. Suppl. 216 (2011) 69 [arXiv:1103.3919] [InSPIRE].

[41] S. Chandrasekhar, The mathematical theory of black holes, Int. Ser. Monogr. Phys. 69, Oxford University Press, Oxford U.K. (1983).

[42] B.C. da Cunha and F. Novaes, Kerr-de Sitter greybody factors via isomonodromy, arXiv: 1508.04046 [INSPIRE].

[43] N.J. Hitchin, G.B. Segal and R.S. Ward, Integrable systems: twistors, loop groups, and Riemann surfaces, Oxford University Press, Oxford U.K. (2013).

[44] H. Stephani, D. Kramer, M. MacCallum, C. Hoenselaers and E. Herlt, Exact solutions of Einstein's field equations, Cambridge University Press, Cambridge U.K. (2003). 